
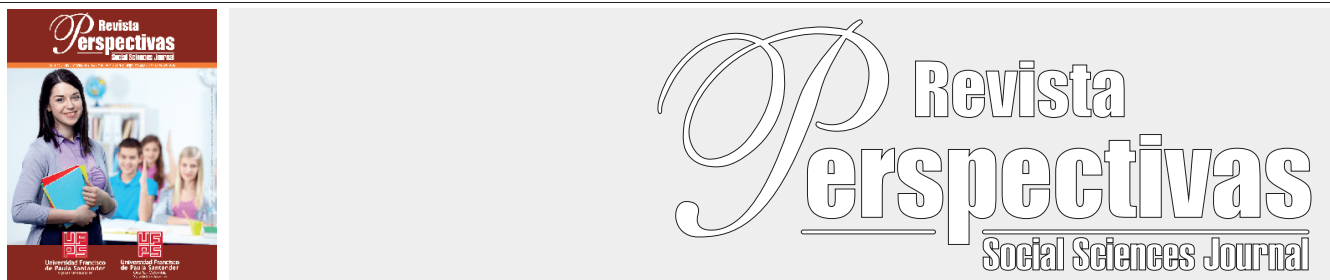

Artículo Original

https://doi.org/10.22463/25909215.1867

\title{
Representaciones sociales en jóvenes escolarizados: una lectura desde la sexualidad
}

Social representations in young students: a reading from sexuality

Jennys Yecenia Yances-Padilla ${ }^{1 *}$, Isabel Alicia Sierra-Pineda ${ }^{2}$

${ }^{1 *}$ Candidata a Doctora en Ciencias de la Educación, jyances18@hotmail.com, ORCID 0000-0002-3627-1908, Funcionaria Secretaría de la Mujer, Montería, Colombia.

2Doctora en Psicología y Educación, iasierrapineda@correo.unicordoba.edu.co, ORCID 0000-0002-2699-8230 Universidad de Córdoba, Montería, Colombia.

Cómo citar: J. Yances-Padilla y I. Sierra-Pineda, "Representaciones sociales en jóvenes escolarizados: una lectura desde la sexualidad". Perspectivas, vol. 4, no. 2, 6-12.

Recibido: Junio 27, 2018; Aprobado: Octubre 03, 2018.

\begin{tabular}{|c|c|}
\hline & RESUMEN \\
\hline \multirow[t]{2}{*}{$\begin{array}{l}\text { Palabras clave: } \\
\text { Cuerpo } \\
\text { Poder } \\
\text { Representaciones sociales } \\
\text { Sexualidad }\end{array}$} & $\begin{array}{l}\text { La teoría de las representaciones sociales no se interesa solo por indagar los conceptos o las estructuras } \\
\text { cognitivas de los sujetos, por el contrario, su interés se focaliza en investigar cómo determinadas prácticas } \\
\text { sociales permiten la configuración de esa representación y se fundamenta en la configuración de las vivencias y } \\
\text { experiencias de los actores en contextos particulares. El presente artículo presenta los resultados de una amplia } \\
\text { investigación que pretendió identificar las representaciones que tienen jóvenes escolarizados sobre sexualidad } \\
\text { en tres instituciones de educación media en la ciudad de Montería - Colombia. El horizonte metodológico } \\
\text { se estructuró desde el enfoque cualitativo - hermenéutico y se utilizó como técnicas de recolección de la } \\
\text { información el grupo focal. Los resultados evidencian que gran parte de las representaciones sociales de los } \\
\text { participantes hacen énfasis en temáticas como la planificación familiar, el embarazo precoz, la prevención de } \\
\text { enfermedades sexualmente transmisibles, el cuidado de la salud y el machismo como elemento predominante. }\end{array}$ \\
\hline & ABSTRACT \\
\hline $\begin{array}{l}\text { Body } \\
\text { Power } \\
\text { Social representations } \\
\text { Sexuality }\end{array}$ & $\begin{array}{l}\text { The theory of social representations is not only interested in investigating the concepts or cognitive structures } \\
\text { of the subjects, on the contrary, their interest is focused on investigating how certain social practices allow } \\
\text { the configuration of that representation and is based on the configuration of experiences and experiences of } \\
\text { the actors in particular contexts. This article presents the results of extensive research that sought to identify } \\
\text { the representations that young people who have been educated about sexuality have in three institutions of } \\
\text { secondary education in the city of Montería - Colombia. The methodological horizon was structured from the } \\
\text { qualitative - hermeneutical approach and the focus group was used as information gathering techniques. The } \\
\text { results show that a large part of the social representations of the participants emphasize issues such as family } \\
\text { planning, early pregnancy, prevention of sexually transmitted diseases and health care. }\end{array}$ \\
\hline
\end{tabular}

\section{Introducción}

Se considera que la sexualidad es un elemento esencial en la formación del ser humano; esta última, representa el pilar fundamental para el buen ejercicio de la convivencia en sociedad, entendida esta actividad como un ejercicio más allá de la simple posibilidad de tomar decisiones en el ámbito público, debido a que implica la posibilidad que tienen mujeres y hombres de tomar decisiones autónomas sobre su propio cuerpo en los campos de la sexualidad y la reproducción.
En este sentido, las representaciones sociales que, sobre sexualidad se han venido construyendo entre la población juvenil, hace pensar que motivan la existencia de relaciones coitales y entorpecen el goce natural de las mismas, desconociendo el ejercicio responsable de sus derechos sexuales y sus derechos reproductivos (Cardona, Ariza y Gaona, 2015; Monroy, 2002), el cual, se ha convertido en un paradigma de convivencia que propende por el desarrollo integral de las personas, garantizando el desarrollo libre, sano, seguro y satisfactorio de la sexualidad y la reproducción. 
En respuesta a esto, a lo largo del tiempo, se han implementado campañas de prevención en salud sexual y reproductiva por parte de los gobiernos nacional, regional y local, sin embargo los resultados no han sido los más eficientes; esto se debe a que, a pesar de la existencia de una política en este campo, aún no se pasa a la acción y a la práctica en el ofrecimiento de la información y los servicios que se requieren para la disminución de las condiciones desfavorables en los grupos poblacionales más necesitados. A esto se le suma que de acuerdo a la encuesta de la Asociación Probienestar de la Familia Colombiana - Profamilia (2005), la población joven es una de las más vulnerables, especialmente aquella que está fuera del sistema escolar.

Ante la panorámica descrita, surge el cuestionamiento sobre el papel que han cumplido la familia y las instituciones educativas en la educación sexual de los y las jóvenes. En Colombia, desde la Ley General de Educación (Ley 115 de 1994) y su decreto reglamentario 1860 de agosto del mismo año, se establece el desarrollo de la educación sexual como proyecto pedagógico obligatorio, que, realizado de manera transversal, debe estar articulado al Proyecto Educativo Institucional (PEI). Es decir, desde la escuela, se debía garantizar un aporte para la solución de la problemática descrita para los jóvenes, pues, su intención era generar una propuesta de transformación y construcción de la cultura sexual a nivel de afectos, conocimientos, actitudes, comportamientos y valores de su comunidad.

De otro modo, el presente estudio se fundamenta en la teoría de las representaciones sociales y ve en la sexualidad un foco representacional de investigación que se aborda ante la existencia de un conjunto de signos que abarcaran todas las esferas del ser humano, en su parte intelectual, social y religiosa, sería necesario acuñarle el concepto de antropológico de Cultura (Palabra conflictiva que ha estado sujeta a múltiples cambios semánticos a través del tiempo). No obstante, esta palabra ha evolucionado y pasó de ser un simple término que designaba, para los romanos, un trabajo específico con la tierra, a ser un concepto de suma valoración en los actuales tiempos.

Un aspecto de gran importancia en la existencia y el desarrollo de las comunidades es la cultura, ya que, en ella, se consignan las vivencias, hábitos, valores y costumbres. Por eso, todos estos aspectos generan influencia sobre los procesos mentales de las personas pertenecientes a un mismo grupo social, sin embargo, por su condición fluctuante al avance, cambia con el tiempo. Al generarse este cambio, también se generan transformaciones en las formas de pensar de la colectividad en un momento dado, es decir, las representaciones. Pues lo que piensa dicha colectividad, es el resultado de la interacción y la vivencia de sus valores culturales, hecho que establece a la cultura y las representaciones como elementos inalienables.

Para comprender cabalmente este proceso y la categorización conceptual, se definirá Representación Social, como un término que se relaciona con la sociología, la psicología, y cuyos orígenes han establecido en la cultura y en las visiones de mundo. Este concepto se ha empleado principalmente en las Ciencias Sociales, aunque actualmente ha sido de provecho para otras disciplinas a las que no escapa la psicología.

A través de estas representaciones sociales, se construyen ideologías que definen la forma de pensar de los integrantes de un grupo social determinado. En este sentido, Mora (2002) citando a Moscovici (1979) plantea que:

La representación social es una modalidad particular del conocimiento, cuya función es la elaboración de los comportamientos y la comunicación entre los individuos. Es un corpus organizado de conocimientos y una de las actividades psíquicas gracias a las cuales los hombres hacen inteligible la realidad física y social, se integran en un grupo o en una relación cotidiana de intercambios, liberan los poderes de su imaginación... son sistemas de valores, nociones y prácticas que 
proporciona a los individuos los medios para orientarse en el contexto social y material, para dominarlo. (p. 7)

Es decir, al hablar de representación social, se hace referencia al conocimiento con el que las personas cuentan para definir su forma de pensar y sus actuaciones en situaciones específicas, un elemento determinante en la representación social son las creencias, las cuales se establecen como mecanismos consientes o inconscientes producidos por el cúmulo de saberes existentes, por lo que Rokeach (1968) afirma que las creencias son grandes presunciones acerca de uno mismo y de la realidad física y social.

\section{Materiales y Métodos}

El presente estudio se enmarca en el enfoque cualitativo puesto que es una opción que no se agota exclusivamente a una dimensión filosófica, sino que trasciende a una propuesta metodológica de comprensión de la realidad social bajo la metáfora de un texto susceptible de ser interpretado y para el efecto desarrolla en sus distintas acepciones y caminos metodológicos con particularidades muy propias que la hacen distinta a otras alternativas de investigación (Sandoval, 1997)

De esta manera, el paradigma cualitativo hace posible indagar el por qué lo social toma determinada forma (Bonilla y Rodríguez, 2005) e intenta hacer una aproximación global de las situaciones sociales para explorarlas, escribirlas y comprenderlas. Por lo tanto, posibilita un tipo de conocimiento que se construye en el diálogo, la interacción, la intersubjetividad y la cotidianidad. En ese sentido, los estudios de orden cualitativo reivindican las realidades subjetiva e intersubjetiva como objetos legítimos de conocimiento científico y la vida cotidiana, como el escenario básico de construcción, constitución y desarrollo de los distintos planos que configuran e integran dichas realidades como dimensiones específicas del mundo humano.
El horizonte metodológico de la investigación se abordó desde las propuestas epistemológicas de la hermenéutica, que en la versión de Ricoeur (1987) se define como la teoría de las reglas que gobiernan una exégesis, es decir, una interpretación de un texto particular o una colección de signos susceptible de ser considerada como un texto. En esta perspectiva, uno de los conceptos básicos es el de círculo hermenéutico que describe el movimiento entre la forma de ser del intérprete y el ser que es revelado por el texto.

La recolección de la información ser realizó utilizando la técnica de grupos focales como forma de interacción colectiva entre el investigador y un número determinado de sujetos en un espacio común (Gamboa, Lago y Hernández, 2016) para recolectar datos sobre la construcción colectiva de significados. El grupo focal según Duarte citado por Gamboa, Vargas y Hernández (2017):

Es una técnica de tipo cualitativo y por lo tanto no implica establecer muestras representativas; tampoco se requiere una se- lección aleatoria de los participantes; sin embargo, es necesario emplear una estrategia para definir la composición del grupo. El grupo debe elegirse a partir de los supuestos teóricos que reflejen aquellos segmentos de la población que pueden suministrar in- formación significativa en términos del objetivo que se persigue. (p. 14)

La muestra del estudio se determinó según el criterio de intencionalidad, convocando a 35 estudiantes de los grados 10 y 11 que brindaran información amplia y suficiente para realizar el estudio. La identificación de los estudiantes se muestra a manera de códigos como E1 (estudiante 1) y G1 (grupo focal 1). En la siguiente tabla I se evidencia la muestra y las instituciones donde se llevó a cabo la investigación:

Tabla 1. Participantes del estudio

\begin{tabular}{cccc}
\hline $\begin{array}{c}\text { NOMBRE DE LA } \\
\text { INSTITUCIÓN }\end{array}$ & $\begin{array}{c}\text { NÚMERO DE } \\
\text { ESTUDIANTES }\end{array}$ & $\begin{array}{c}\text { GÉNERO } \\
\text { M }\end{array}$ & $\begin{array}{c}\text { GÉNERO } \\
\text { F }\end{array}$ \\
\hline $\begin{array}{c}\text { Institución Educativa } \\
\text { Marceliano Polo }\end{array}$ & 8 & 4 & 4 \\
$\begin{array}{c}\text { Institución Educativa } \\
\text { Gimnasio Lucila Zapata } \\
\begin{array}{c}\text { Arenas } \\
\text { Institución Educativa los } \\
\text { Córdobas }\end{array}\end{array}$ & 17 & 11 & 6 \\
\hline
\end{tabular}




\section{Resultados y discusiones}

En el presente apartado se muestran los resultados y discusión teórica de los relatos que surgieron de la interacción dialógica del investigador y los sujetos de investigación donde surgen categorías como sexualidad, sociedad y poder.

El significado de la sexualidad para los estudiantes parte de una asociación con las relaciones íntimas de pareja, pero se reconoce la existencia de diversos componentes, entre los cuales se destacan valores o principios como el respeto, sinceridad y la confianza cuando manifiestan que:

La sexualidad es como un acto de intimidad entre una pareja, es como una relación que tiene muchos componentes. (E1, G2) Yo creo que ahí va incluido el respeto, la confianza, el diálogo. En la sexualidad se tiene que dialogar mucho para poder llegar a un consenso de que es lo que se quiere hacer y qué es lo que no se quiere hacer, que es lo que esa persona desea desde su interior hacer y compartirlo a la otra persona y que lo apruebe. Sí, son como ciertos valores que se deben tener sobre la mesa cuando se empieza una relación para llegar a esa sexualidad, a esa sexualidad abierta entre ellos, porque son dos personas, no son tres ni cuatro, entonces ahí juega mucho la confianza (E2, $\mathrm{G} 2$ )

Es conveniente aclarar que estas declaraciones provienen de estudiantes pertenecientes al género femenino, de tal forma que se observa una asociación de la sexualidad con el componente afectivo, expresado a través de términos como aprecio, cariño, lo cual representa una justificación social y cultural para el desarrollo de relaciones sexuales (Giordano, Longmore, \& Manning, 2006).

De otro lado, la sexualidad se asocia con uno de los componentes propuestos por el MEN (2008), el concepto de identidad de género; se aprecia una mayor profundidad en la percepción de esta dimensión, la cual supera el nivel de lo puramente físico para adentrarse en los dominios de la autonomía del individuo, esto lo manifiestan:

pues yo pienso y veo la sexualidad no solamente como algo de pareja, yo pienso que eso también va desde el ser, desde la forma en que nos reconozcamos desde nuestro género, parte netamente desde ahí, desde el hecho de que yo soy mujer y me reconozco como mujer. Me gustan los hombres, pero no solamente es desde el hecho de tener intimidad con una persona, sino que es más amplio. (E3, G2)

Puede contrastarse esta declaración con la definición propuesta por el MEN (2008) para la identidad sexual de una persona señalando que la "identidad de género hace referencia al sentimiento profundo de una persona de igualdad, unidad y persistencia como hombre, mujer o ambivalente, a través del tiempo y a pesar de los cambios físicos o psíquicos" (p.11).

Finalmente, existe una representación de la sexualidad que involucra factores limitantes $o$ coercitivos, especialmente el componente moral, que asocia la actividad sexual del individuo con lo patológico; en el caso de presentarse conductas sexuales inadecuadas o incorrectas que se salgan de los parámetros de lo que se considera normal o permitido, se percibe la enfermedad o las problemáticas derivadas como un castigo, así lo afirman:

Es un tema que a nadie le gusta hablar. Yo tomo la sexualidad como un complemento donde usted acuerda en un pacto de confianza entre una mujer y un hombre, en el cual usted entrega afecto para a veces procrear o mantener una relación. En muchos casos, nosotros los jóvenes vemos la sexualidad como una diversión, cuando uno comprende las causas y los tipos de consecuencias que puede traer (E2, G4). 
En este sentido, el filósofo francés Michel Foucault en su análisis de la sexualidad como un objeto de dominación por parte de las estructuras de poder, identifica los procesos de regulación como una de las estrategias más importantes para controlar y disciplinar los cuerpos y las conductas. Es de esta forma como se generan discursos reguladores que provienen de diferentes instituciones sociales como la iglesia, la familia, la escuela y el estado. Además, los discursos referentes a la sexualidad cubren un rango muy amplio: políticos, morales, médicos, biológicos, pedagógicos, etc. (García, 2009).

La sexualidad como precepto moral ha sido utilizado como instrumento de dominación desde la antigüedad, normalizando los comportamientos de los individuos y estableciendo conductas que permiten regular el disfrute de los placeres. Estas prescripciones morales encuentran uno de sus mejores aliados en componentes de la estructura social que ejercen poder, como el que representa la iglesia, cuyo pensamiento reaccionario ha hecho presencia a lo largo de la historia, aprovechándose de la ignorancia y el temor que suscitan en los individuos. De esta forma, los discursos reguladores configuran la construcción de subjetividades y los individuos son sometidos a los dictados de las estructuras de poder que dominan en un contexto determinado.

Asimismo, para los estudiantes, la sexualidad en el núcleo familiar y en la sociedad en general se asume como una temática prohibida, como una verdad incómoda, que debe ocultarse. Además, la sexualidad se representa desde una mirada reduccionista en la cual predomina la dimensión biológica, estas afirmaciones se evidencian cuando los jóvenes señalan que:

Yo creo que una de las cosas para que eso suceda es un poco del tabú que existe, eh porque, digamos ir a una droguería y comprar un condón no es algo fácil, da mucha vergüenza (risas), y no solo con eso, con cualquier otro método, una mujer comprar eso es un tabú, también el tabú de hablar con los padres, no más del mismo padre, el padre hablar con el hijo de como son las cosas y eso da mucha vergüenza, da mucha pena. (E4, G2)

En Historia de la Sexualidad II, El uso de los placeres, Foucault (1987) describe la manera como la sexualidad y los placeres fueron convertidos en un problema moral y estudia su evolución desde la Antigüedad Clásica hasta la Modernidad. Existe una notable diferencia entre la forma como fue asumida esta problemática por la cultura griega, en la cual la ética de la sexualidad estaba conformada por un conjunto de principios morales que enfatizaban en el cuidado de sí mismo y en lo que Foucault denomina las "técnicas del Yo". Por el contrario, para el cristianismo, la ética sexual estaba ligada a códigos de estricto cumplimiento y a la austeridad; la asociación del placer sexual con lo pecaminoso y su prohibición a través de la formulación de reglas de conducta es una característica que contrasta con la ética un tanto más permisivo del paganismo propio de la cultura griega.

Esta genealogía de la ética sexual desarrollada por Michel Foucault, permite concluir que la influencia del cristianismo en las sociedades occidentales está basada en la consideración de las conductas sexuales como un objeto de estudio que debe cumplir de manera estricta códigos de conducta establecidos.

Esta formación de individuos que obedecen leyes morales de obligatorio cumplimiento, que se fundamenta en el miedo, que considera los placeres sexuales como un pecado y que promueve la represión del deseo y la abstinencia propios del sistema moral del cristianismo, que ha moldeado la cultura occidental y ha contribuido a la construcción de subjetividades heterónomas, confusas, contradictorias, sometidas a las estructuras de poder. En este sentido los estudiantes afirman que:

Yo creo que en las casas utilizamos una doble moral, porque cuando un niño ve una película de una guerra no sé qué, 
donde matan a las personas es normal, pero cuando ven una escena obscena entonces, es tápese los ojos, entonces es enseñándole al niño que eso está mal, que está como oculto, que es prohibido y es lo que genera la desinformación, pero también la curiosidad. (E2, G2). Por lo general los hombres tienen muchas mujeres (risas) y las publicaciones de hombres son más de mujeres desnudas, por ejemplo, una mujer no puede tener esas imágenes en el celular, eso demuestra machismo (E3, G2)

El análisis de las complejas interrelaciones que se establecen entre los individuos, la sexualidad, la ética y las relaciones de poder conduce a las estrategias y procedimientos que han sido desarrollados históricamente para el control y regulación de las conductas sexuales, especialmente a partir de la modernidad. La consideración del cuerpo como un dispositivo de poder y la creación de tecnologías orientadas a la dominación de las poblaciones humanas constituye uno de los resultados más relevantes del pensamiento de Foucault (1977), y demuestra precisamente lo que narran los sujetos desde sus propias vivencias.

\section{Conclusiones}

Se logran evidenciar discursos importantes que en cierto modo se contrastan con los enunciados presentes en las políticas públicas de educación sexual, con categorías similares como por ejemplo la prevención. Al respecto, en los relatos de los estudiantes se evidencia de una forma clara esta perspectiva que hace énfasis en temáticas como la planificación familiar, embarazo precoz, prevención de enfermedades sexualmente transmisibles, cuidado de la salud, entre otros.

De otro modo, en la representación que tienen los estudiantes, la sexualidad surge como una asociación con las relaciones íntimas de pareja, pero se reconoce la presencia de múltiples componentes, entre los cuales se destacan valores o principios como el respeto, sinceridad y la confianza.

Un elemento que hay que rescatar en los discursos de los estudiantes de género femenino es la asociación de la sexualidad con el componente afectivo, expresado a través de términos como aprecio, cariño, lo cual representa una justificación social y cultural para el desarrollo de relaciones sexuales.

\section{Referencias}

Asociación Probienestar de la Familia Colombiana (Profamilia). (2005). Saluid sexual y reproductiiva. Resultados Encuesta Nacional de Demografía y Salud. Bogotá, Colombia: Printex Impresores Ltda.

Bonilla, E., \& Rodríguez, P. (2005). Más Allá de los dilemas de los métodos. Bogotá: Norma.

Cardona, D, Ariza-Gerena, A., Gaona-Restrepo, C., \& Medina-Pérez, Ó. (2015). Conocimientos sobre sexualidad en adolescentes escolares en la ciudad de Armenia, Colombia. Revista Archivo Médico de Camagüey, 19(6), 568576. Recuperado de http://scielo.sld.cu/ scielo.php?script $=$ sci_arttext\&pid $=\mathrm{S} 1025$ $02552015000600003 \& \operatorname{lng}=$ es\&tlng=es

Decreto $\mathrm{N}^{\circ}$ 1860. (1994) Por el cual se reglamenta parcialmente la Ley 115 de 1994, en los aspectos pedagógicos y organizativos generales. Diario Oficial No 41.473 de la República de Colombia. Foucault, M. (1977). Historia de la sexualidad I. La voluntad de saber. México: Siglo XXI.

Foucault, M. (1987). Historia de la sexualidad II. London: Penguin Books.

Gamboa, A., Lago, D., \& Hernández, F. (2016). Calidad dela docencia universitaria comprensión de los discursos y políticas institucionales de una universidad pública en norte de Santander. Saber, ciencia y libertad, 11(1), 197-210. Obtenido de https:/dialnet.unirioja.es/servlet/ articulo? codigo $=5847004$

Gamboa-Suárez,A., Vargas-Toloza, R., \&HernándezSuárez, C. (2017). Investigación formativa: 
Pretensiones curriculares y representaciones sociales de actores educativos en el programa de Enfermería de la Universidad Francisco de Paula Santander-Cúcuta. Colombia. Universidad y Salud, 19(1), 60-66. Obtenido de http:// www.scielo.org.co/scielo.php? script $=$ sci arttext\&pid=S0124-71072017000100060

García, M. (2009). El señor Foucault y eso que se dio en llamar sexualidad. Tramas, 32,31-62.

Giordano, P., Longmore, M., \& Manning, W. (2006). Gender and the meanings of adolescent romantic relationships: A focus on boys. American Sociological Review, 71(2), 260-287.

Ministerio de Educación Nacional. (2008). Programa Nacional de Educación para la Sexualidad y construcción de Ciudadanía. La dimensión de la sexualidad. en la educación de nuestros niños, niñas, adolescentes y jóvenes. Colombia.

Ley 115. (1994). Por la cual se expide la ley general de educación. El Congreso Nacional de la República de Colombia: Bogotá. Obtenido de https://www.mineducacion.gov.co/1621/ articles-85906_archivo_pdf.pdf

Monroy, A. (2002). Salud y sexualidad en la adolescencia y juventud: guía práctica para padres y educadores. México: Editorial Pax.

Moscovici, S. (1979). El psicoanálisis, su imagen y su público. Buenos Aires. Huemul.

Mora, M. (2002). La teoría de las representaciones sociales de Serge Moscovici. Athenea, (2), 1-25. Recuperado de https://www.raco.cat/index. php/Athenea/article/viewFile/34106/33945

Ricoeur, P. (1987). Freud: una interpretación de la cultura. México: Siglo Veintiuno Editores.

Rokeach, M. (1968). A theory of organization and change within value-attitude systems. Journal of Social Issues, 24(1), 13-33.

Sandoval, C. (1997). Investigación cualitativa. Programa de Especialización en teoría, métodos y técnicas de investigación social. Bogotá: ICFES. 